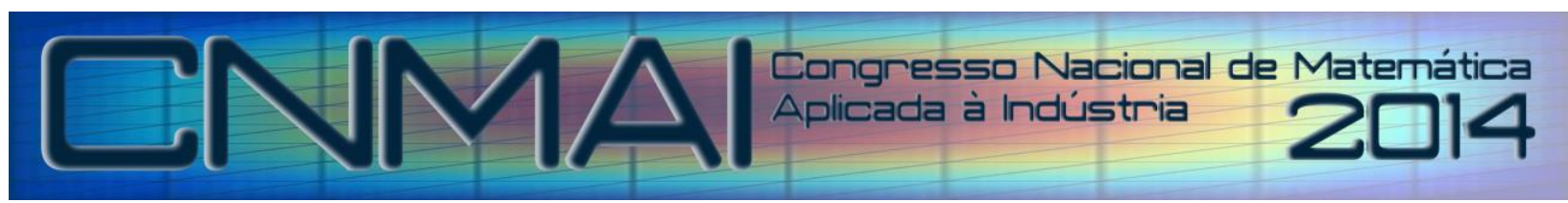

18 a 21 de novembro de 2014, Caldas Novas - Goiás

\title{
MODELO DE PROGRAMAÇÃO LINEAR INTEIRA PARA O PROBLEMA DE CARREGAMENTO DE MÚLTIPLOS CONTÊINERES COM RESTRIÇÕES DE CARREGAMENTO COMPLETO DE GRUPO DE ITENS E DE ESTABILIDADE VERTICAL
}

\author{
Cleder Marcos Schenekemberg, cledercms@ hotmail.com ${ }^{1}$ \\ Deidson Vitorio Kurpel, kurpeld@gmail.com ${ }^{1}$ \\ Cassius Tadeu Scarpin, cassiusts@ufpr.br ${ }^{1}$ \\ ${ }^{1}$ Universidade Federal do Paraná - Programa de Pós-Graduação em Métodos Numéricos em Engenharia, Centro \\ Politécnico da UFPR - Curitba - Paraná/Brasil
}

\begin{abstract}
Resumo: Este trabalho trata um caso particular dos problemas de corte e empacotamento, conhecido como Problema de Carregamento de Contêiner. O objetivo de estudo deste trabalho é apresentar um modelo de programação linear inteira capaz de assegurar o carregamento completo de grupo de itens e a estabilidade vertical da carga num conjunto limitado de contêineres. Embora comuns no cotidiano, algumas dessas considerações práticas raramente são tratadas em trabalhos correlatos. Testes computacionais com instâncias adaptadas da literatura foram realizados para validar o modelo proposto. Os resultados mostram que a abordagem é coerente e descreve adequadamente as situações abordadas.
\end{abstract}

Palavras-chave: Otimização Combinatória, Problema de Carregamento de Contêiner, Carregamento Completo de Grupo de Itens, Estabilidade Vertical.

\section{INTRODUÇÃO}

O Problema de Carregamento de Contêineres (Container Loading Problem - CLP) representa, junto à Pesquisa Operacional, um caso particular dos problemas de corte e empacotamento. Para resolvê-lo, procura-se dispor caixas retangulares ortogonalmente de tal modo que a ocupação do volume nos contêineres disponíveis seja maximizada. Qualquer arranjo de caixas no interior de um contêiner é denominado padrão de carregamento. É importante assegurar que todas as caixas estão colocadas inteiramente dentro de um contêiner e não exista sobreposição de caixas. A melhor ocupação do espaço interno ou a minimização do uso de contêineres podem reduzir substancialmente o preço do transporte de mercadorias, impactando o preço final dos produtos e a relação entre compradores e revendedores.

No entanto, o CLP conta com uma série de características que devem ser levadas em conta em sua resolução. Orientação de caixas, estabilidade da carga, distribuição de peso dentro dos contêineres, múltiplos destinos de entrega são algumas das doze considerações práticas listadas por Bischoff e Ratcliff (1995) ao se carregar um contêiner e, assim sendo, podem ser levadas em conta ao se propor um algoritmo que busque resolver o CLP.

Dentre os trabalhos efetuados nesta área, destacamos Tsai, Malstrom e Kuo (1993), Mohanty, Mathur e Ivancic (1994), Bischoff e Ratcliff (1995), Chen, Lee e Shen (1995), Padberg (2000), Eley (2003), Moura e Oliveira (2009), Almeida e Figueiredo (2010), Ren, Tian e Sawaragi (2011) e Junqueira, Morabito e Yamashita (2012). Bortfeldt e Wäscher (2013) afirmam que, embora nos últimos anos o número de publicações que tratam do problema de carregamento de contêineres tenha aumentado, ainda há considerações práticas pouco estudadas.

Este trabalho apresenta uma proposta de resolução do problema de carregamento de múltiplos contêineres idênticos com restrições de carregamento completo de grupo de itens e estabilidade vertical, baseada em programação linear inteira. A mesma procura alocar completamente um dado conjunto de caixas em um ou mais contêineres idênticos, de modo a otimizar o uso do espaço de cada contêiner, garantindo um percentual mínimo de apoio para cada caixa.

O artigo está organizado da seguinte maneira: na seção 2, o problema de carregamento de múltiplos contêineres idênticos com restrições de carregamento completo e estabilidade vertical é explicado em detalhes, juntamente com um 
modelo de programação linear inteira para descrevê-lo; na seção 3, os resultados computacionais obtidos na avaliação do modelo utilizando conjuntos de teste da literatura são apresentados e analisados; finalmente, na seção 4 são apresentadas as principais conclusões alcançadas, bem como perspectivas para trabalhos futuros.

\section{DESCRIÇÃO DO PROBLEMA E FORMULAÇÃO DO MODELO MATEMÁTICO}

O Problema de Carregamento de Contêineres pode ser divido em dois subproblemas. No primeiro, denominado Problema de Carregamento de Bins (Bin Packing Problem - BPP), o espaço de armazenagem é suficiente para a alocação de todas as caixas, visto que o número de contêineres é, em geral, irrestrito. Logo, busca-se minimizar o número de contêineres necessários para carregar todos os itens. Já no segundo, chamado Problema da Mochila Tridimensional (Three Dimensional Knapsack Problem - 3DKP), o espaço de um número limitado de contêineres não é suficiente para armazenar todos os itens. O objetivo é, então, determinar um subconjunto de caixas que maximiza a utilização do volume dos contêineres ou valor associado à carga.

De acordo com a tipologia de Wäscher, Haußner e Schumann (2007), o modelo matemático proposto neste artigo trata do problema de Multi-Mochilas Idênticas (Multiple Identical Knapsack Problem - MIKP), no qual os contêineres são idênticos, isto é, possuem as mesmas dimensões e, consequentemente, o mesmo volume.

\subsection{Modelo Matemático e Restrição de Estabilidade Vertical}

Segundo Junqueira, Morabito e Yamashita (2012) a restrição prática de estabilidade pode ser dividida em dois casos: estabilidade horizontal e estabilidade vertical. Em síntese, procura-se oferecer sustentação da face inferior das caixas, no caso de estabilidade vertical, ou no suporte das faces laterais, quando se trata de estabilidade horizontal. Por simplicidade, este artigo aborda apenas a estabilidade vertical, por meio de um parâmetro $\alpha \epsilon[0,1]$, proposto por Eley (2002), que define o percentual mínimo de estabilidade vertical que deve ser considerado no carregamento de contêiner.

O modelo proposto é uma extensão para o MIKP da modelagem para carregamento de um único contêiner apresentada por Junqueira, Morabito e Yamashita (2012). Um total de $C$ contêineres idênticos, $C \geq 1$, cujas largura, comprimento e altura são, respectivamente, $L, W$ e $H$, devem ser carregados com $m$ tipos de caixas distintas. Cada caixa do tipo $i, i=1, \ldots, m$, que deve ser alocada em um contêiner $k$, onde $k=1, \ldots, C$, possui largura $l_{i}$, comprimento $w_{i}$, altura $h_{i}$ e volume $v_{i}$. Além disso, o problema é restrito no que diz respeito à quantidade de caixas disponíveis para carregamento, isto é, existe um total de $b_{i}$ caixas do tipo $i$ que podem ser colocadas no interior dos contêineres.

Tomando o sistema de coordenadas cartesiano, seja $(p, q, r)$ o vértice inferior frontal esquerdo de uma caixa do tipo $i$ no interior de um contêiner $k$ (veja Fig. 1). Os conjuntos abaixo, definidos por Junqueira, Morabito e Yamashita (2012), indicam as possíveis localizações que a caixa pode assumir em relação às dimensões de cada contêiner, considerando ainda que as caixas possuam orientação fixa, isto é, a largura, comprimento e altura das caixas são paralelas às respectivas dimensões do contêiner:

$$
\begin{array}{ccc}
X=\left\{p \mid 0 \leq p \leq L-\min _{i}\left(l_{i}\right),\right. & p \in \mathbb{Z}, & i=1, \ldots, m\} \\
Y=\left\{q \mid 0 \leq q \leq W-\min _{i}\left(w_{i}\right),\right. & q \in \mathbb{Z}, & i=1, \ldots, m\} \\
Z=\left\{r \mid 0 \leq r \leq H-\min _{i}\left(h_{i}\right),\right. & r \in \mathbb{Z}, & i=1, \ldots, m\}
\end{array}
$$

Embora os elementos dos conjuntos acima restrinjam a números inteiros, essa condição pode ser facilmente relaxada. As variáveis de decisão do modelo são variáveis binárias definidas como:

$$
x_{i k p q r}=\left\{\begin{array}{cc}
\begin{array}{c}
\text { Se uma caixa do tipo } i \text { tem seu vértice inferior frontal } \\
\text { esquerdo no ponto }(p, q, r) \text { do contêiner } k, \text { tal que }
\end{array} \\
0 \leq p \leq L-l_{i}, & 0 \leq q \leq W-w_{i}, \quad 0 \leq r \leq H-h_{i}
\end{array}\right.
$$

Considere também os conjuntos:

$$
\begin{gathered}
X_{i}=\left\{p \in X \mid 0 \leq p \leq L-l_{i}\right\} \\
Y_{i}=\left\{q \in Y \mid 0 \leq q \leq W-w_{i}\right\} \\
Z_{i}=\left\{r \in Z \mid 0 \leq r \leq H-h_{i}\right\}
\end{gathered}
$$

O Problema de Multi-Mochilas Idênticas (MIKP) é formado a partir do modelo binário de Junqueira, Morabito e Yamashita (2012) da seguinte maneira: 


$$
\max \sum_{k=1}^{C} \sum_{i=1}^{m} \sum_{p \in X_{i}} \sum_{q \in Y_{i}} \sum_{r \in Z_{i}} v_{i} \cdot x_{i k p q r}
$$

Sujeito a:

$$
\begin{gathered}
\sum_{i=1}^{m} \sum_{\left\{p \in X_{i} \mid\right.} \sum_{\left.s-l_{i}+1 \leq p \leq s\right\}} \sum_{\left\{q \in Y_{i} \mid\right.} \sum_{\left.t-w_{i}+1 \leq q \leq t\right\}} \sum_{\left\{r \in Z_{i} \mid\right.} x_{i k p q r} \leq 1, \quad k=1, \ldots, C \\
\sum_{k=1}^{C} \sum_{\left.p \in X_{i}+1 \leq r \leq u\right\}} \sum_{q \in Y_{i}} \sum_{r \in Z_{i}} x_{i k p q r} \leq b_{i}, \quad i=1, \ldots, m \\
\sum_{\left\{i \mid r^{\prime}-h_{i} \geq 0\right\}}^{m} \sum_{\left\{p \in X_{i} \mid p^{\prime}-l_{i}+1 \leq p \leq p^{\prime}+l_{j}-1\right\}} L_{\left\{q \in Y_{i} \mid q^{\prime}+w_{i}+1 \leq q \leq q^{\prime}+w_{j}-1\right\}} \cdot W_{i j} \cdot x_{i k p q\left(r^{\prime}-h_{i}\right)} \geq \alpha \cdot l_{j} \cdot w_{j} \cdot x_{j k p^{\prime} q^{\prime} r^{\prime}} \\
k=1, \ldots, C
\end{gathered}
$$

Onde

$$
\begin{gathered}
\left\{\begin{array}{l}
L_{i j}=\min \left(p+l_{i}, p^{\prime}+l_{j}\right)-\max \left(p, p^{\prime}\right) \\
W_{i j}=\min \left(q+w_{i}, q^{\prime}+w_{j}\right)-\max \left(q, q^{\prime}\right)
\end{array}\right. \\
x_{i k p q r} \in\{0,1\}, \quad i=1, \ldots, m, \quad k=1, \ldots, C, \quad p \in X_{i}, \quad q \in Y_{i}, \quad r \in Z_{i} \\
s \in X, \quad t \in Y, \quad u \in Z, \quad p^{\prime} \in X_{j}, \quad q^{\prime} \in Y_{j}, \quad r^{\prime} \in Z_{j}^{*}, \quad j=1, \ldots, m
\end{gathered}
$$

No modelo acima, a função objetivo, representada pela Eq. (8) maximiza o volume das caixas alocadas nos $C$ contêineres disponíveis; a Eq. (9) evita a sobreposição de caixas nos contêineres, impedindo a ocupação de um ponto $(s$, $t, u)$ qualquer no interior de uma caixa cujo vértice inferior frontal esquerdo é o ponto $(p, q, r)$ por uma caixa distinta; a Eq. (10) não permite o carregamento de caixas do tipo $i$ além da disponibilidade máxima; a Eq. (11) assegura que uma porcentagem mínima da área da base das caixas está apoiada por outras caixas (ou pelo piso do contêiner); por fim, a Eq. (12) define o domínio das variáveis do modelo.

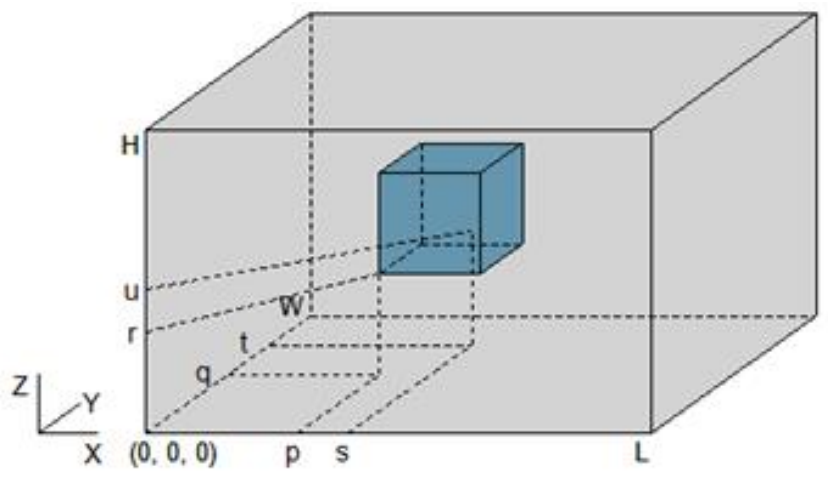

Figura 1. Uma caixa do tipo $i$ no interior de um contêiner $k$. Fonte: Junqueira, Morabito e Yamashita (2012).

\subsection{Restrição de Carregamento Completo de Grupo de Itens}

Em alguns casos, é necessário garantir que um subconjunto de caixas seja integralmente carregado (ou deixado de fora do carregamento), mesmo que isso ocasione o desperdício de parte do volume útil do contêiner. Isto é, se uma caixa pertencente a determinado subconjunto é carregada, todas as demais também devem ser transportadas ou, de modo alternativo, se uma caixa não é empacotada, todas as demais também devem ser deixadas de lado. Tais subconjuntos serão chamados, ao longo deste trabalho, de grupos de itens ou simplesmente, grupos.

Para Bischoff e Ratcliff (1995), em situações que envolvem o carregamento completo de grupo de itens há a exigência de que todas as caixas fazem parte de uma entidade funcional (grupos de itens contendo peças de um produto eletrônico, por exemplo) devem estar presentes no padrão de carregamento. De acordo com Eley (2003), a restrição de carregamento completo pode ser dividida em quatro variantes distintas: (i) um grupo composto por um único tipo de caixa deve ser completamente empacotado; (ii) um grupo constituído por uma combinação de caixas de diferentes tipos deve fazer parte do padrão de carregamento; (iii) o número de caixas de um único tipo deve ser múltiplo de determinado grupo composto por caixas do mesmo tipo; (iv) uma combinação de caixas de tipos distintos deve ser múltiplo de um grupo de itens formado pelos mesmos tipos de caixas. Este artigo trata da restrição de carregamento completo do tipo 
(iii); se, por exemplo, um grupo é formado por sete caixas de mesmo tipo, os modelos propostos buscam garantir que o total de caixas carregadas desse tipo seja um múltiplo de sete. Vale ressaltar que, no modelo proposto neste trabalho, as caixas que compõe um dado grupo não precisam, necessariamente ser carregadas no mesmo contêiner, isto é, caixas de um grupo podem ser alocadas em diferentes contêineres desde que todas as caixas do grupo façam parte do padrão de carregamento.

Seja um grupo de itens composto apenas por caixas do tipo $i$. Se $\zeta_{i}$ é a quantidade de caixas do grupo, então a restrição de carregamento completo na qual são enviados $y_{i}$ grupos de itens formados por caixas do tipo $i$ pode ser expressa da seguinte maneira:

$$
\begin{gathered}
\sum_{k=1}^{C} \sum_{p \in X_{i}} \sum_{q \in Y_{i}} \sum_{r \in Z_{i}} x_{i k p q r}=\zeta_{i} \cdot y_{i} \\
y_{i} \in \mathbb{Z}_{+}, \quad 1 \leq i \leq m
\end{gathered}
$$

A Eq. (13) garante que a quantidade de caixas alocadas no interior dos contêineres é múltipla do total de caixas que formam o grupo, enquanto a Eq. (14) define o domínio das variáveis presentes na restrição.

\section{EXPERIMENTOS COMPUTACIONAIS}

Os testes computacionais foram realizados em três cenários distintos. No primeiro, os modelos foram formados através da Eq. (8)-(10) e da Eq. (12), isto é, considerando apenas restrições de não-sobreposição e limitação de caixas. O segundo cenário, com a Eq. (8)-(12), foi elaborado com o acréscimo da restrição de estabilidade vertical definida pela Eq. (11) aos modelos do caso anterior. Quando a restrição de carregamento completo apresentada na seção 2.2 é incorporada ao modelo matemático da seção 2.1, obtém-se o último cenário, formado pela Eq. (8)-(14). Os modelos foram gerados por meio de um algoritmo em linguagem de programação Microsoft Visual Studio Ultimate 2012@ (versão 11.0.50727.1), e resolvidos utilizando-se o software CPLEX@ Interactive Optimizer (versão 12.5.1) utilizando parâmetros padrão do mesmo. Os testes foram efetuados em um computador com sistema operacional Microsoft Windows 7, processador Intel@ Core ${ }^{T M}$ i3 de $2.53 \mathrm{GHz}$, memória RAM de 4 GB e sistema operacional de 32 bits. Não foi estabelecido um limite de tempo para execução dos testes.

Os modelos foram avaliados utilizando uma adaptação dos conjuntos de testes propostos por Mohanty, Mathur e Ivancic (1994). Um dos contêineres em cada um dos conjuntos originais foi escolhido através de um processo aleatório de seleção. A quantidade disponível dos mesmos foi estipulada como o dobro da disponibilidade original do contêiner escolhido. As dimensões, os tipos e a disponibilidade das caixas não sofreram alterações. Os conjuntos de testes adaptados estão sumarizados na Tab. 1.

Para considerar a restrição de carregamento completo, optou-se por utilizar a mesma metodologia empregada por Eley (2003), criando dois grupos de itens que devem ser completamente carregados: o primeiro é formado por $7 \%$ do total das caixas disponíveis do tipo 1, enquanto o segundo é composto por $12 \%$ da totalidade de caixas do tipo 2 . Nos casos em que o cálculo da porcentagem retornava um número não inteiro, a parte fracionária do valor foi descartada e uma unidade foi adicionada à parte inteira do resultado inicial, de modo que a quantidade de caixas seja inteira em todos os grupos de itens. Para os modelos que possuem restrição de estabilidade vertical, a constante de estabilidade foi definida em $\alpha=1$, isto é, a face inferior de cada caixa carregada deve estar totalmente apoiada nas demais caixas ou no piso do contêiner.

A Tabela 2 apresenta um resumo da quantidade de variáveis e restrições presentes em cada um dos modelos gerados para teste. Alguns modelos não puderam ser gerados em razão de insuficiência de memória computacional; estas situações são representadas na tabela através do símbolo “_”.

O problema de carregamento de múltiplos contêineres idênticos, proposto na Eq. (8)-(10) e Eq. (12), possui em sua formulação apenas restrições de não sobreposição e limitação da quantidade de caixas, as quais são compostas unicamente por variáveis binárias. Similar ao modelo de Junqueira, Morabito e Yamashita (2012), percebe-se que, à medida que há um aumento na variedade de caixas, ou quando as mesmas possuem dimensões pequenas em relação aos contêineres, ocorre um aumento no número de restrições de cada modelo. Aqui, chama a atenção o papel desempenhado pela quantidade de contêineres disponíveis para carregamento; note que há um considerável aumento no número de restrições de não sobreposição quando mais contêineres são considerados (compare os conjuntos 2 e 7). Os modelos propostos nas Eq. (8)-(12) possuem a mesma totalidade de variáveis binárias e restrições do modelo da Eq. (8)-(10) e Eq. (12); entretanto, o acréscimo da restrição de estabilidade vertical ao modelo faz aumentar significativamente o número de restrições (veja os conjuntos 2 e 15). 
TABELA 1. Conjuntos de teste adaptados de Mohanty, Mathur e Ivancic (1994).

\begin{tabular}{|c|c|c|c|c|c|c|c|c|c|c|}
\hline \multirow{3}{*}{$\begin{array}{l}\text { Conjunto } \\
\text { de Teste }\end{array}$} & \multicolumn{5}{|c|}{ Caixas } & \multicolumn{5}{|c|}{ Contêineres } \\
\hline & \multirow{2}{*}{$\begin{array}{c}\text { Tipo } \\
i \\
\end{array}$} & \multirow{2}{*}{$\begin{array}{c}\text { Disponibilidade } \\
b_{i}\end{array}$} & \multicolumn{3}{|c|}{ Dimensões } & \multirow{2}{*}{$\begin{array}{c}\text { Disponibilidade } \\
K\end{array}$} & \multicolumn{3}{|c|}{ Dimensões } & \multirow{2}{*}{$\begin{array}{c}\begin{array}{c}\text { Volume } \\
\text { Total dos } \\
\text { Contêineres }\end{array} \\
V T\end{array}$} \\
\hline & & & $l_{i}$ & $w_{i}$ & $h_{i}$ & & $L$ & $W$ & $H$ & \\
\hline \multirow{2}{*}{1} & 1 & 20 & 2 & 6 & 8 & \multirow{2}{*}{4} & \multirow{2}{*}{14} & \multirow{2}{*}{14} & \multirow{2}{*}{14} & \multirow{2}{*}{10976} \\
\hline & 2 & 50 & 8 & 4 & 10 & & & & & \\
\hline \multirow{4}{*}{2} & 1 & 45 & 8 & 16 & 4 & & & & & \\
\hline & 2 & 60 & 8 & 8 & 8 & 10 & 20 & 16 & 20 & 64000 \\
\hline & 3 & 50 & 8 & 12 & 12 & 10 & 20 & 10 & 20 & 04000 \\
\hline & 4 & 25 & 12 & 4 & 4 & & & & & \\
\hline & 1 & 35 & 10 & 5 & 5 & & & & & \\
\hline 3 & 2 & 40 & 5 & 15 & 10 & 2 & 20 & 15 & 25 & 15000 \\
\hline & 3 & 28 & 5 & 15 & 15 & & & & & \\
\hline 4 & 1 & 50 & 36 & 28 & 24 & 10 & 40 & 36 & 52 & 748800 \\
\hline 4 & 2 & 60 & 40 & 32 & 20 & 10 & 40 & 30 & 52 & 148800 \\
\hline & 1 & 25 & 15 & 20 & 25 & & & & & \\
\hline 5 & 2 & 30 & 10 & 15 & 10 & 4 & 35 & 25 & 40 & 140000 \\
\hline & 3 & 40 & 20 & 25 & 35 & & & & & \\
\hline & 1 & 28 & 18 & 10 & 8 & & & & & \\
\hline 6 & 2 & 37 & 12 & 14 & 10 & 10 & 18 & 16 & 20 & 57600 \\
\hline & 3 & 30 & 6 & 10 & 8 & & & & & \\
\hline & 1 & 15 & 7 & 4 & 10 & & & & & \\
\hline 7 & 2 & 12 & 3 & 5 & 11 & 2 & 20 & 15 & 29 & 17400 \\
\hline & 3 & 20 & 6 & 9 & 12 & & & & & \\
\hline & 1 & 20 & 6 & 8 & 11 & & & & & \\
\hline & 2 & 17 & 5 & 15 & 9 & & & & & \\
\hline 8 & 3 & 13 & 4 & 13 & 7 & 4 & 17 & 16 & 20 & 21760 \\
\hline 8 & 4 & 24 & 12 & 13 & 8 & 4 & 11 & 10 & 20 & 21100 \\
\hline & 5 & 21 & 10 & 14 & 11 & & & & & \\
\hline & 6 & 80 & 8 & 2 & 4 & & & & & \\
\hline & 1 & 18 & 10 & 13 & 11 & & & & & \\
\hline 9 & 2 & 20 & 8 & 9 & 10 & 2 & 40 & 22 & 26 & 45760 \\
\hline 9 & 3 & 12 & 7 & 12 & 15 & 2 & 40 & 22 & 20 & 45700 \\
\hline & 4 & 22 & 14 & 15 & 11 & & & & & \\
\hline & 1 & 50 & 2 & 4 & 16 & & & & & \\
\hline 10 & 2 & 70 & 2 & 8 & 16 & 2 & 16 & 16 & 16 & 8192 \\
\hline & 3 & 80 & 8 & 2 & 4 & & & & & \\
\hline & 1 & 30 & 5 & 11 & 14 & & & & & \\
\hline 11 & 2 & 28 & 9 & 12 & 10 & 2 & 19 & 15 & 26 & 14820 \\
\hline 11 & 3 & 37 & 7 & 13 & 17 & 2 & 19 & $1 J$ & 20 & 14020 \\
\hline & 4 & 23 & 10 & 10 & 8 & & & & & \\
\hline & 1 & 35 & 3 & 6 & 8 & & & & & \\
\hline 12 & 2 & 40 & 5 & 9 & 4 & 2 & 20 & 18 & 12 & 8640 \\
\hline & 3 & 15 & 8 & 9 & 12 & & & & & \\
\hline 13 & 1 & 56 & 7 & 8 & 3 & 4 & 18 & 15 & 9 & 9770 \\
\hline 13 & 2 & 28 & 12 & 11 & 4 & 4 & 10 & $1 J$ & 9 & 9720 \\
\hline & 1 & 25 & 4 & 8 & 6 & & & & & \\
\hline 14 & 2 & 32 & 10 & 12 & 6 & 4 & 20 & 20 & 10 & 16000 \\
\hline & 3 & 45 & 14 & 14 & 8 & & & & & \\
\hline & 1 & 25 & 6 & 6 & 9 & & & & & \\
\hline 15 & 2 & 25 & 12 & 10 & 8 & 4 & 28 & 14 & 18 & 28224 \\
\hline 15 & 3 & 15 & 4 & 7 & 5 & 4 & 20 & 14 & 10 & 28224 \\
\hline & 4 & 20 & 7 & 9 & 11 & & & & & \\
\hline & 1 & 35 & 15 & 18 & 17 & & & & & \\
\hline 16 & 2 & 30 & 11 & 14 & 11 & 2 & 48 & 70 & 32 & 245040 \\
\hline & 3 & 25 & 24 & 26 & 15 & & & & & \\
\hline
\end{tabular}


Quando os modelos passam a considerar a restrição de carregamento completo, por meio da adição da Eq. (13)-(14) ao proposto pelas Eq. (8)-(12), o número de variáveis binárias permanece inalterado em relação ao problema de carregamento de múltiplos contêineres idênticos sem carregamento completo. Este fato já era esperado, uma vez que, como não houve nenhuma alteração nas dimensões dos contêineres, também não deve existir nenhuma mudança na quantidade e na formulação das restrições de não sobreposição. Entretanto, como nos conjuntos de testes a quantidade de caixas dos tipos 1 e 2 a serem empacotadas deve ser múltiplo do total de caixas que compõe os grupos de itens, isto adiciona duas variáveis inteiras positivas aos problemas testados. $\mathrm{O}$ acréscimo de restrições de carregamento completo ocorre na mesma ordem, isto é, se há $g$ grupos de itens, o modelo (8)-(14) terá, em sua formulação, $g$ restrições deste tipo.

Tabela 2. Número de restrições e de variáveis dos modelos propostos.

\begin{tabular}{|c|c|c|c|c|c|c|}
\hline \multirow{2}{*}{$\begin{array}{c}\text { Conjunto de } \\
\text { Teste }\end{array}$} & \multicolumn{2}{|c|}{$\mathrm{N}^{\circ}$. de Variáveis } & \multicolumn{4}{|c|}{$\mathrm{N}^{\circ}$. de Restrições } \\
\hline & Binárias & Inteiras & $\begin{array}{c}\text { Não } \\
\text { Sobreposição }\end{array}$ & $\begin{array}{c}\text { Disponibilidade } \\
\text { de Caixas }\end{array}$ & $\begin{array}{c}\text { Estabilidade } \\
\text { Vertical }\end{array}$ & $\begin{array}{c}\text { Carregamento } \\
\text { Completo }\end{array}$ \\
\hline 1 & 4816 & 2 & 4004 & 2 & 4040 & 2 \\
\hline 2 & 43160 & 2 & 28730 & 4 & 40040 & 2 \\
\hline 3 & 5946 & 2 & 7392 & 3 & 5640 & 2 \\
\hline 4 & 14700 & 2 & 14850 & 2 & 14200 & 2 \\
\hline 5 & - & - & - & - & - & - \\
\hline 6 & 15050 & 2 & 11830 & 3 & 13860 & 2 \\
\hline 7 & 18024 & 2 & 8640 & 3 & 17082 & 2 \\
\hline 8 & - & - & - & - & - & - \\
\hline 9 & - & - & - & - & - & - \\
\hline 10 & 4170 & 2 & 5850 & 3 & 3240 & 2 \\
\hline 11 & 6506 & 2 & 3420 & 4 & 6070 & 2 \\
\hline 12 & 5480 & 2 & 4212 & 3 & 4432 & 2 \\
\hline 13 & 3528 & 2 & 2688 & 2 & 3004 & 2 \\
\hline 14 & 6988 & 2 & 4420 & 3 & 5512 & 2 \\
\hline 15 & 27444 & 2 & 12600 & 4 & 24948 & 2 \\
\hline 16 & - & - & - & - & - & - \\
\hline
\end{tabular}

A Tabela 3 apresenta um sumário dos resultados obtidos após a realização dos testes. Em alguns casos, o computador não possuía memória suficiente para gerar o modelo pelo Microsoft Visual Studio Ultimate 2012@. Assim, não há informações relevantes quanto à porcentagem de ocupação dos contêineres nem quanto ao tempo de execução. Tal como na Tab. 2, o símbolo “-” foi empregado nestes casos.
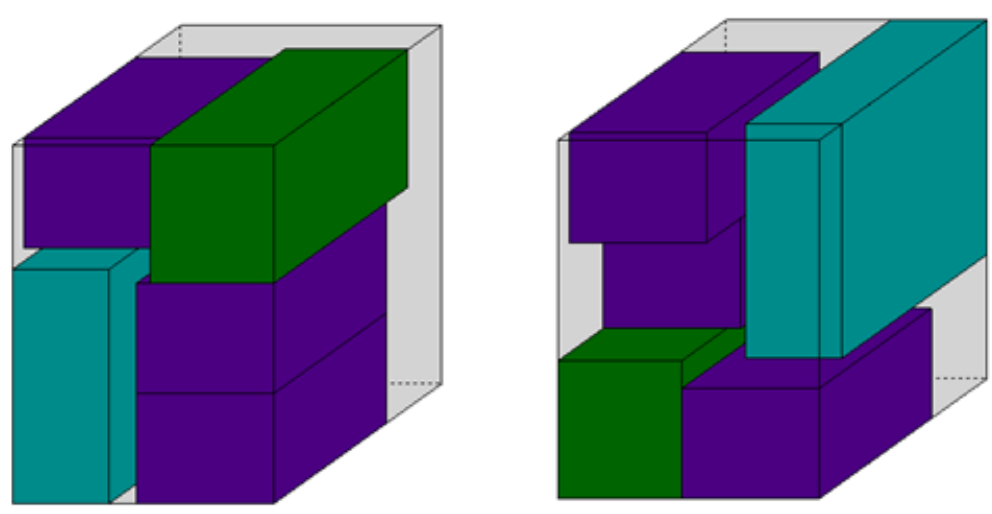

Figura 2. Padrão de carregamento do conjunto de teste 11 obtido pelo modelo da Eq. (8)-(10) e Eq. (12).

Uma breve análise mostra que, nos modelos com a Eq. (8)-(10) e Eq. (12) a ocupação média do volume disponível dos contêineres foi de $73,70 \%$, com desvio padrão de $18,72 \%$. Esses valores são próximos aos obtidos com os modelos com estabilidade vertical $(73,29 \%$ e $18,91 \%$, respectivamente) e com os modelos com restrição de estabilidade e carregamento de grupos $(72,89 \%$ e $18,82 \%$, respectivamente). 
Tabela 3. Panorama dos testes computacionais efetuados como os modelos propostos.

\begin{tabular}{|c|c|c|c|c|c|c|c|c|c|}
\hline \multirow{2}{*}{$\begin{array}{c}\text { Conjunto de } \\
\text { Teste }\end{array}$} & \multicolumn{3}{|c|}{$\begin{array}{c}\text { Sem Carregamento Completo e } \\
\text { Sem Estabilidade Vertical - } \\
\text { Eq. (8)-(10) e Eq. (12) }\end{array}$} & \multicolumn{3}{|c|}{$\begin{array}{c}\text { Sem Carregamento Completo e } \\
\text { Com Estabilidade Vertical - } \\
\text { Eq. (8)-(12) }\end{array}$} & \multicolumn{3}{|c|}{$\begin{array}{c}\text { Com Carregamento Completo e } \\
\text { Com Estabilidade Vertical - } \\
\text { Eq. (8)-(14) }\end{array}$} \\
\hline & $\begin{array}{c}\text { Função } \\
\text { Objetivo }\end{array}$ & $\begin{array}{c}\text { Ocupação } \\
(\mathrm{em} \%)\end{array}$ & $\begin{array}{c}\text { Tempo } \\
\text { (s) }\end{array}$ & $\begin{array}{l}\text { Função } \\
\text { Objetivo }\end{array}$ & $\begin{array}{c}\text { Ocupação } \\
(\text { em \%) }\end{array}$ & $\begin{array}{c}\text { Tempo } \\
\text { (s) }\end{array}$ & $\begin{array}{c}\text { Função } \\
\text { Objetivo }\end{array}$ & $\begin{array}{c}\text { Ocupação } \\
(\text { em \%) }\end{array}$ & $\begin{array}{c}\text { Tempo } \\
(\mathrm{s})\end{array}$ \\
\hline 1 & 5760 & 52,48 & 0,45 & 5760 & 52,48 & 0,17 & 5760 & 52,48 & 0,22 \\
\hline 2 & 52800 & 82,50 & 293,13 & 52800 & 82,50 & 84,29 & 52800 & 82,50 & 100,09 \\
\hline 3 & 15000 & 100,00 & 10,13 & 15000 & 100,00 & 2,34 & 15000 & 100,00 & 2,34 \\
\hline 4 & 512000 & 68,38 & 17,77 & 512000 & 68,38 & 1,12 & 506368 & 67,62 & 1,22 \\
\hline 5 & - & - & - & - & - & - & - & - & - \\
\hline 6 & 43200 & 75,00 & 5,34 & 43200 & 75,00 & 1,83 & 43200 & 75,00 & 1,81 \\
\hline 7 & 11436 & 65,72 & 54,52 & 11436 & 65,72 & 14,27 & 11436 & 65,72 & 10,64 \\
\hline 8 & - & - & - & - & - & - & - & - & - \\
\hline 9 & - & - & - & - & - & - & - & - & - \\
\hline 10 & 8192 & 100,00 & 3,60 & 8192 & 100,00 & 1,50 & 8192 & 100,00 & 1,67 \\
\hline 11 & 10054 & 67,84 & 3,09 & 9328 & 62,94 & 2,59 & 9120 & 61,54 & 6,40 \\
\hline 12 & 8640 & 100,00 & 2,29 & 8640 & 100,00 & 1,98 & 8496 & 98,33 & 39,97 \\
\hline 13 & 4224 & 43,46 & 0,44 & 4224 & 43,46 & 0,78 & 4224 & 43,46 & 0,84 \\
\hline 14 & 9600 & 60,00 & 1,78 & 9600 & 60,00 & 0,50 & 9600 & 60,00 & 0,50 \\
\hline 15 & 19476 & 69,01 & 32,51 & 19476 & 69,01 & 472,85 & 19209 & 68,06 & 830,74 \\
\hline 16 & - & - & - & - & - & - & - & - & - \\
\hline Média & & 73,70 & 35,42 & & 73,29 & 48,69 & & 72,89 & 83,04 \\
\hline Desvio Padrão & & 18,72 & 82,78 & & 18,91 & 135,67 & & 18,82 & 237,25 \\
\hline
\end{tabular}

Quanto ao tempo necessário para a resolução dos modelos temos que, na Eq. (8)-(10) e Eq. (12) o tempo médio de resolução foi de 35,42 segundos, com desvio padrão de 82,78 segundos. Enquanto que, para os modelos da Eq. (8)-(12) e Eq. (8)-(14) o tempo médio decorrido foi de 48,69 segundos (desvio padrão de 135,67) e 83,04 segundos (com desvio padrão de 237,25), respectivamente. Note que, embora tenha obtido o menor tempo médio, o primeiro cenário obteve tempo superior em 8 dos 12 testes realizados (em destaque na Tab. 3). Vale destacar a expressiva contribuição do conjunto de teste 15 no aumento do tempo médio do segundo e terceiro cenários. Contudo, percebe-se, em geral, que o acréscimo das restrições práticas de estabilidade vertical e carregamento completo de grupos não afetou substancialmente o tempo de resolução dos modelos propostos.

As Figuras 2, 3 e 4 mostram, respectivamente, o padrão de carga obtido para o conjunto de teste 11 com os modelos das Eq. (8)-(10) e Eq. (12), Eq. (8)-(12) e Eq. (8)-(14). As caixas foram coloridas de acordo com os tipos de itens: (i) tipo 1 - roxo; (ii) tipo 2 - verde; (iii) tipo 3 - azul claro; (iv) tipo 4 - azul escuro. Cabe lembrar que, para os modelos com restrição de carregamento completo as caixas do tipo 1 e 2 formam grupos de caixas. De acordo com a formação de grupos proposta por Eley (2003), temos que a quantidade de caixas do grupo 1 é $\zeta_{1}=3$, enquanto para o grupo 2 $\zeta_{2}=4$.

O padrão de carga apresentado na Fig. 2, embora que não possua estabilidade vertical, conta com a maior ocupação do volume total dos contêineres disponíveis. Este fato só reforça o esperado, pois este é o cenário que possui a menor quantidade de considerações práticas. Por outro lado, apesar de uma menor ocupação do volume disponível dos contêineres, o padrão de carga apresentado na Fig. 3 apresenta estabilidade vertical em todas as caixas do carregamento.
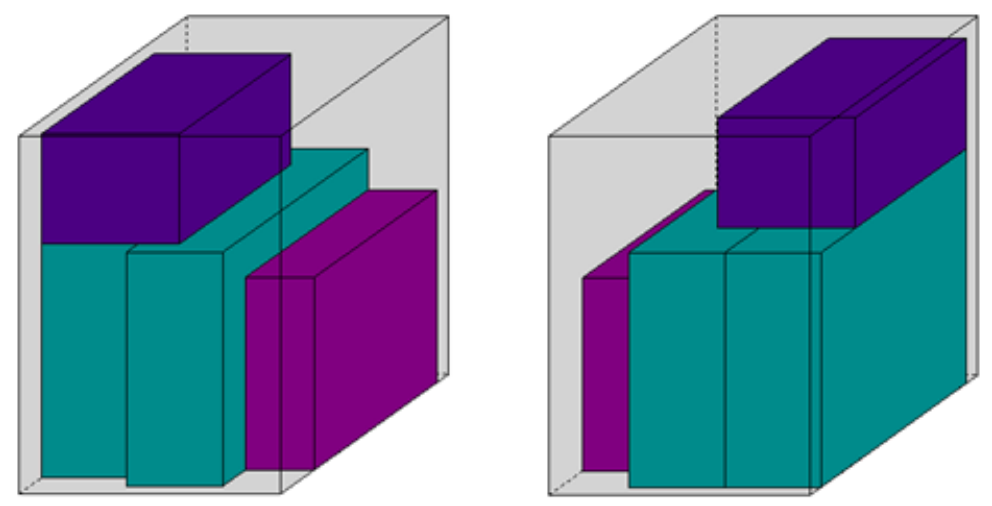

Figura 3. Padrão de carregamento do conjunto de teste 11 obtido pelo modelo da Eq. (8)-(12). 
Dos três cenários testados, o padrão de carga apresentado na Fig. 4 obteve a menor ocupação do volume disponível (confira a Tab. 3). Este cenário, composto pelos grupos 1 e 2, carregou apenas caixas do grupo 2 (4 unidades, ou seja, multiplicidade do conjunto igual a um) e exemplares de caixas do tipo 4 (6 unidades). Percebe-se que a exigência de carregamento de múltiplos de $\zeta_{1}$ e $\zeta_{2}$ diminui substancialmente a quantidade de caixas do tipo $i=3$ (verifique a Fig. 4), acarretando num melhor aproveitamento do espaço dos contêineres quando a restrição de carregamento completo de grupos de itens não é considerada no modelo (Fig. 2 e Fig. 3).

Em vista da grande demanda de memória computacional para geração dos modelos e realização dos cálculos das possíveis posições que as caixas podem assumir ao serem empacotadas nos contêineres, alguns conjuntos de teste não puderam ser avaliados. Assim, a modelagem apresentada neste trabalho pode ter dificuldades para tratar de problemas reais, que envolvem diversos contêineres de mesma dimensão e variados tipos de caixas. Entretanto, vale ressaltar que, como apontado na Tab. 2, os modelos testados possuem um alto número de variáveis e restrições, sendo bastante trabalhosa a tarefa de encontrar uma solução adequada para os mesmos.
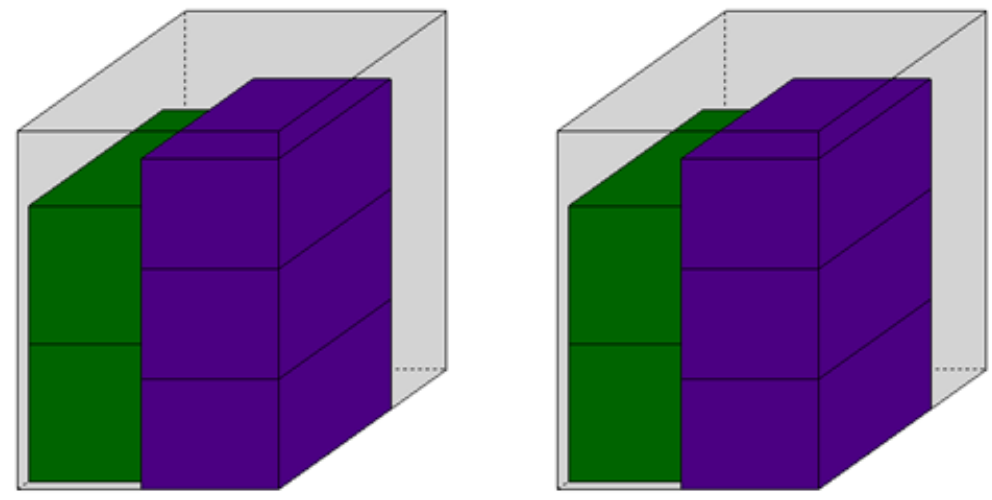

Figura 4. Padrão de carregamento do conjunto de teste 11 obtido pelo modelo da Eq. (8)-(14).

\section{CONSIDERAÇÕES FINAIS}

Este trabalho apresentou uma proposta de resolução do problema de carregamento de múltiplos contêineres idênticos com restrições de estabilidade vertical e carregamento completo de grupo de itens, baseada em programação linear inteira. O objetivo da mesma é alocar completamente um dado conjunto de caixas de distintos tamanhos em contêineres idênticos, de modo e otimizar o uso do espaço de cada contêiner, garantindo que certo subconjunto de caixas se faça, obrigatoriamente, presente no padrão de carregamento.

O software CPLEX@ Interactive Optimizer foi utilizado para efetuar testes computacionais com dados adaptados da literatura de carregamento de contêineres. Os resultados obtidos mostraram que os modelos propostos representam corretamente as considerações práticas tratadas, embora a otimalidade seja alcançada apenas em modelos de tamanho moderado, em virtude da necessidade de avaliar as possíveis posições de cada caixa nos contêineres disponíveis.

Futuramente, este trabalho pode ser utilizado como motivação para pesquisas que abordem as demais variações de restrição de carregamento completo citadas por Eley (2003), e que tratem de situações mais realistas, trabalhando com as demais considerações práticas apontadas por Bischoff e Ratcliff (1995) e considerem contêineres de diferentes dimensões.

\section{REFERÊNCIAS}

ALMEIDA, A.; FIGUEIREDO, M. B. 2010. A particular approach for the three-dimensional packing problem with additional constraints. Computers \& Operations Research, v. 37, n. 11, p. 1968-1976.

BISCHOFF, E. E.; RATCLIFF, M. S. W. 1995. Issues in the development of approaches to container loading. Omega, v. 23 , n. 4 , p. $377-390$.

BORTFELDT, A.; WÄSCHER, G. 2013. Constraints in container loading - A state-of-the-art review. European Journal of Operational Research, v. 229, n. 1, p. 1-20.

CHEN, C.S.; LEE, S. M.; SHEN, Q.S. 1995.An analytical model for the container loading problem. European Journal of Operational Research, v. 80, n. 1, p. 68-76.

ELEY, M. 2002. Solving container loading problems by block arrangement. European Journal of Operational Research, v. 141, n. 2, 393-409.

ELEY, M. 2003. A bottleneck assignment approach to the multiple container loading problem. OR Spectrum, v. 25, n. 1, p. 45-60. 
JUNQUEIRA, L.; MORABITO, R.; YAMASHITA, D. S. 2012. Three-dimensional container loading models with cargo stability and load bearing constraints. Computer \& Operations Research, v. 39, n. 1, p. 74-85.

MOHANTY, B. B.; MATHUR, K.; IVANCIC, N. J. 1994. Value considerations in three-dimensional packing - A heuristic procedure using the fractional knapsack problem. European Journal of Operational Research, v. 74, n. 1, p. $143-151$.

MOURA, A., OLIVEIRA, J.F. 2009. An integrated approach to the vehicle routing and container loading problems. OR Spectrum, v. 31, n. 4, p. 775-800.

PADBERG, M. 2000. Packing small boxes into a big box. Mathematical Methods of Operations Research, v. 52, n. 1, p. $1-21$.

REN, J.; TIAN, Y.; SAWARAGI, T. 2011. A tree search method for the container loading problem with shipment priority. European Journal of Operational Research, v. 214, n. 3, p. 526-535.

TSAI, R.D.; MALSTROM, E. L.; KUO, W. 1993. Three dimensional palletization of mixed box sizes. IIE Transactions, v. 25, n. 4, p. 64-75.

WÄSCHER, G., HAU $\beta N E R$, H., SCHUMANN, H. 2007. An improved typology of cutting and packing problems. European Journal of Operational Research, v. 183, n. 3, p. 1109-1130.

\title{
RESPONSABILIDADE AUTORAL
}

"Os autores são os únicos responsáveis pelo conteúdo deste trabalho".

\section{INTEGER LINEAR PROGRAMMING MODEL FOR MULTIPLE CONTAINER LOADING PROBLEM WITH COMPLETE SHIPMENT AND VERTICAL STABILITY CONSTRAINTS}

\author{
Cleder Marcos Schenekemberg, cledercms@ hotmail.com ${ }^{1}$ \\ Deidson Vitorio Kurpel, kurpeld@gmail.com ${ }^{1}$ \\ Cassius Tadeu Scarpin, cassiusts@ufpr.br ${ }^{1}$ \\ ${ }^{1}$ Universidade Federal do Paraná - Programa de Pós-Graduação em Métodos Numéricos em Engenharia, Centro \\ Politécnico da UFPR - Curitba - Paraná/Brazil
}

\begin{abstract}
This work deals with a particular case of the cutting and packing problems, known as Container Loading Problem. This paper aims to present a integer linear programming model that ensures the complete shipment of a group of items and the vertical stability of the load in a limited set of containers. Although common in daily situations, some of these practical considerations are seldom addressed in related works. Computational tests on instances adapted from the literature were performed to validate the proposed model. The results show that the approach is consistent and appropriately describes the situations addressed.
\end{abstract}

Keywords: Combinatorial optimization, Container loading problem, Complete Shipment of group of items, Vertical Stability. 